\title{
INTEGRATION OF MACHINE LEARNING AND COMPUTATIONAL FLUID DYNAMICS TO DEVELOP TURBULENCE MODELS FOR IMPROVED TURBINE WAKE MIXING PREDICTION
}

\author{
Harshal D. Akolekar* \\ Yaomin Zhao \\ Richard D. Sandberg \\ Department of Mechanical Engineering \\ University of Melbourne, VIC, 3010, Australia \\ Email: hakolekar@student.unimelb.edu.au
}

\author{
Roberto Pacciani \\ Department of Industrial Engineering, \\ University of Florence, Italy \\ roberto.pacciani@unifi.it
}

\begin{abstract}
This paper presents development of accurate turbulence closures for wake mixing prediction by integrating a machine-learning approach with Reynolds Averaged Navier-Stokes (RANS)-based computational fluid dynamics (CFD). The data-driven modeling framework is based on the gene expression programming (GEP) approach previously shown to generate non-linear RANS models with good accuracy. To further improve the performance and robustness of the data-driven closures, here we exploit that GEP produces tangible models to integrate RANS in the closure development process. Specifically, rather than using as cost function a comparison of the GEP-based closure terms with a frozen high-fidelity dataset, each GEP model is instead automatically implemented into a RANS solver and the subsequent calculation results compared with reference data. By first using a canonical turbine wake with inlet conditions prescribed based on high-fidelity data, we demonstrate that the CFD-driven machine-learning approach produces non-linear turbulence closures that are physically correct, i.e. predict the right downstream wake development and maintain an accurate peak wake loss throughout the domain. We then extend our analysis to full turbine-blade cases and show that the model development is sensitive to the training region due to the presence of deterministic unsteadiness in the near wake region. Models developed including this region have artificially large diffusion coefficients to overcompensate for the vortex shedding steady RANS cannot capture. In contrast, excluding the near wake region in the model development produces the correct physical model behavior, but predictive accuracy in the near-wake remains unsatisfactory. We show that this can be remedied by using the physically consistent models in unsteady RANS, implying that the non-linear closure producing the best predictive accuracy depends on whether it will be deployed in RANS or unsteady RANS calculations. Overall, the models developed with the CFD-assisted machine learning approach were found
\end{abstract}

to be robust and capture the correct physical behavior across different operating conditions.

\section{INTRODUCTION}

The gas turbine, which is the work horse of the aviation industry, has reached a high degree of maturity. However, gas turbine designers continue to strive for even incremental points of efficiency improvements with novel methods, as this can translate to millions of dollars of savings and large reductions in carbon emissions. Current gas turbine design is primarily carried out using Reynoldsaveraged Navier Stokes (RANS) modeling due to their low cost and user-friendliness. However, these calculations lack the required accuracy to reliably advance technology, largely due to the use of a linear stress-strain relation - the Boussinesq approximation [1]. With the increase in the power of computing, high-fidelity simulations are becoming increasingly commonplace but are still not feasible as an iterative industrial design tool. In order to bridge the gap between high and low-fidelity simulations, high-fidelity data sets can be used to extract meaningful physics-based insights and serve as input to machine learning processes that can improve the accuracy of iterative low-fidelity calculations.

Data-driven turbulence modeling has gained momentum, especially in the last five years and is seen by some authors as a promising approach for RANS model development as part of the vision 2030 of CFD [2]. It is quite unlikely that a universal turbulence model will be found using data-driven approaches, but it is conceivable that these models can improve RANS calculations for certain classes of flows. Numerous efforts in trying to improve stress-strain closure relations have been undertaken with machine-learning approaches. Singh et al. [3] used a neural network and a version of the Spalart Allmaras model modified by inverse modeling in order to obtain improved flow prediction across a class of airfoils. A data-driven framework was developed to enhance the RANS mod- 
eled Reynolds stresses for periodic hills and a square duct by Wang et al. [4]. However, even though they improved the Reynolds stress prediction, they could not translate this improvement to better mean flow fields which was due to the models being ill-conditioned. $\mathrm{Wu}$ et al. [5] proposed a method to overcome the ill-conditioning issue, and the developed models not only brought improvement in the mean flow field but also across different test cases. Ling et al. [6] used deep neural networks to improve Reynolds stress predictions for duct flow and flow over a wavy wall. They managed to predict corner vortices for the duct case and flow separation in the wavy wall case; two key flow features that a linear eddy viscosity model could not predict. Data-driven models have not only been developed to improve the Reynolds stress and mean flow predictions, but also to enhance the heat flux modeling. Milani et al. [7] used random forests to improve heat-flux modeling for film cooling applications. Using the simple gradient diffusion hypothesis, random forests were used to infer an improved turbulent diffusivity field. Three distinct data sets related to the jet and cross-flow configuration were used for model development and validation. It was found that the adiabatic effectiveness from the machine-learnt model matched more closely with high-fidelity data than the standard RANS approach. An overview of the existing data-driven methods for turbulence modeling can be found in Duraisamy et al. [8].

Ferreira [9] introduced gene expression programming (GEP), which was a new adaptive algorithm for solving regression based problems. Weatheritt \& Sandberg [10] developed the GEP for turbulence modeling, which generates Galilean invariant explicit algebraic Reynolds stress model (EARSM) closures to improve the stress-strain relation over the Boussinesq approximation. GEP is relatively inexpensive as compared to other machine learning techniques used for previous turbulence model studies. Model development using GEP usually takes of the order of a few core minutes or hours and not of the order of thousands of core hours. The models generated via this method have an added advantage that they do not require any supporting machine learning framework or highfidelity databases for their implementation into computational fluid dynamics (CFD) codes. GEP for turbulence modeling produces tangible symbolic expressions capable of being directly inserted into a RANS solver. These models are physically interpretable, as shown by Akolekar et al. [11], who was able to correlate different models obtained from the training process to physical mechanisms, such as laminar separation and wake-wake interactions. Physics-based information does not remain hidden in the model development process as in the case of neural networks. Over-fitting of data can be avoided by methods such as ensemble averaging, which implies that these models have a robust range of applicability [12]. Wu et al. [13] demonstrated that a number of well established machine-learnt closure development methods can be ill-conditioned: a problem which does not beset the GEP approach.

GEP has been successfully applied to a wide number of turbulence modeling problems and flow scenarios [11, 12, 14-17] to enhance the flow prediction capability of steady, and unsteady RANS (URANS), hybrid RANS / LES (large eddy simulation) and LES. Machine-learnt, non-linear turbulence closures were developed for low pressure turbine (LPT) flows with unsteady inflow conditions using a zonal based model development approach with an aim to enhance the wake mixing prediction for URANS calculations. It was found that the models developed reduced the normalized mean square error of the anisotropy tensor over the Boussinesq approxi- mation by $45 \%$ [11]. Akolekar et al. [12] developed non-linear closure relations which enhanced the wake mixing prediction across two topologically different flows, which is an indicator of the robustness of the models developed. These models were able to reduce the error in peak turbulent kinetic energy (TKE) production relative to direct numerical simulations (DNS) from $70 \%$ (baseline case with Boussinesq approximation) to up to $12 \%$ and also improved mean flow features such as velocity, loss profiles and wake maturity, etc. Schoepplein et al. [18] used GEP to model the subgrid stress of LES for turbulent transport in premixed flames using a DNS data set as a reference. Lav et al. [17] developed a framework using GEP to produce data-driven turbulence models for flows with organized (deterministic or periodic) unsteadiness. Using a triple decomposition, high-fidelity data was split into organized motion and stochastic turbulence. GEP was used to develop closures for the stochastic part of the turbulence which improved the prediction of the stochastic and deterministic regimes of the flow. Recently, Zhao et al. [19] introduced a novel 'CFD-driven' framework for turbulence model development using GEP. It has been used in conjunction with RANS-based CFD to enhance the wake mixing prediction of turbomachinery wakes. They found that this method has high potential for offering robust models.

This study focuses on the development of EARSMs via the newly-developed 'CFD-driven' approach as opposed to the traditional anisotropy-based 'frozen' approach. Of the many physical phenomena that occur in turbines, designers are interested in being able to accurately model the wake mixing using RANS, as this phenomenon governs the stagnation pressure loss in a turbine and also because existing RANS-based turbulence models fail to accurately predict this phenomenon. Therefore, the overall goal of this study is to develop, implement and understand the range of applicability of non-linear EARSMs to improve the wake mixing prediction in LPTs. High-fidelity data sets at realistic engine operating conditions is used as a reference for model generation. Firstly, the method of the 'CFD-driven' approach and the advantages it offers is briefly explained. This approach is then applied to a canonical case of a turbine wake, which has input conditions provided from a direct numerical simulation (DNS), to evaluate whether a model is found that exhibit the correct physical behavior. Like all machine learning analyses, there is great dependence of the models developed on the training regions selected. This work shows in a quantitative sense the effect of selecting or excluding certain portions of the near-wake region for LPTs on the overall model performance and offers physics-based insight. The developed non-linear EARSMs are then tested in a RANS and URANS context for LPTs and conclusions are made on the scope of applicability of the models.

\section{COMPUTATIONAL FLUID DYNAMICS BASED GENE EX- PRESSION PROGRAMMING MODEL DEVELOPMENT}

Method Specifics GEP based EARSM development has been based on the anisotropy-based 'frozen' approach until Zhao et al. [19] integrated CFD calculations with the GEP algorithm. The 'CFD-driven' approach is a method to diversify the way EARSMs are created using GEP. The 'CFD-driven' approach is enabled by the GEP method because GEP returns model equations that are explicit and can be instantly and automatically implemented into RANS solvers during the model development process. With RANS calculations having already been performed in the training loop to evaluate the performance of candidate models suggested by the 
GEP, the resulting 'CFD-driven' models thus are also ready to be implemented into industrial design tools. Another advantage of the 'CFD-driven' training is that the definition of the cost function is more flexible as compared to the 'frozen' approach. Rather than being restricted to quantities that are part of the closure terms such as anisotropy tensors (see Eq. 7), the 'CFD-driven' cost functions can be tailored to capture any important flow feature of interest. One can specify any mean or secondary statistic profiles in order to derive a suitable model. If one does not have large high-fidelity data sets, and only has a few profiles from high-fidelity or experimental data, this approach can still offer suitable models based on this limited data. This approach also ensures that the models generated are numerically stable and realizable as they have already been executed in CFD calculations.

The basic outline of the 'CFD-driven' approach [19] is shown in Fig. 1. The 'CFD-driven' approach creates a population or a large number of candidate models. Unlike the 'frozen' approach,

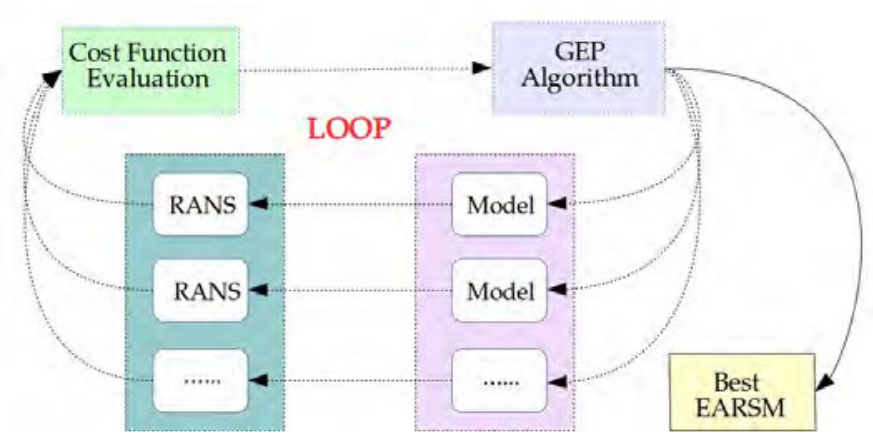

FIGURE 1: The 'CFD-driven' approach using GEP.

wherein the models at each generation are evaluated against a 'frozen' high-fidelity data set, in the 'CFD-driven' approach each of the models that are generated are first run as RANS calculations. For every generation of candidate models, the model equations are read into a RANS solver, and then a series of RANS-based CFD calculations are performed to test different models in parallel. Each of the converged CFD solutions are then evaluated against a cost function based on time-averaged high-fidelity data. The cost function in this case (Eq. 1) can now be based on any flow quantity of interest. Based on the cost function value or fitness of the models, a new generation is formed through GEP evolution. The updated models are evaluated again through integrated CFD calculations. This training process iterates and the population of models evolves. After a number of generations, close to $30-50$ for statistically $2 \mathrm{D}$ LPTs, the best model is found.

Cost Function Since the goal of this work is to enhance the wake mixing prediction, the cost function has to be defined accordingly. It is given as

$$
\begin{aligned}
\Omega(y) & =\frac{P_{t, 1}-P_{t, 2}(y)}{P_{t, 1}-P_{2}}, \\
\Delta_{C} & =\frac{1}{w} \int_{0}^{w}\left(\frac{\Omega^{H i-F i}-\Omega^{R A N S}}{\max \left(\Omega^{H i-F i}\right)}\right)^{2} d y, \\
J^{C F D} & =\Delta_{C 1}+\Delta_{C 2} .
\end{aligned}
$$

where $w$ is the pitch of the cascade. Wake loss profiles from highfidelity data are chosen at two axial locations downstream of the blade TE as targets. From each of the CFD calculations the wake loss profiles are calculated, and the sum of the normalized mean square error of these wake profiles with respect to high-fidelity data is the cost function. Note that the high-fidelity data needed for the computation of the 'CFD-driven' cost function $J^{C F D}$ only needs to include the wake loss profiles $\left(\Omega^{H i-F i}(y)\right)$ at these two locations (not the data from the entire wake region as in the 'frozen' approach). Two axial locations were chosen to evaluate the cost function in order to ensure that the 'CFD-driven' approach predicts the flow correctly across the entire domain. Specifying only one location would ensure that the flow at that location is correctly predicted with no guarantee of other locations. The best model is the one which minimizes the cost function.

Explicit Algebraic Reynolds Stress Models GEP produces EARSMs, whose formulation is provided below. One of the major reasons why RANS-based turbulence models fail to accurately predict wake mixing is due to use of the Boussinesq approximation as a stress-strain relation [1]. It can be broken up into the sum of isotropic and anisotropic contributions

$$
\tau_{i j}=\underbrace{\frac{2}{3} \rho k \delta_{i j}}_{\text {isotropic }}-\underbrace{2 \mu_{t}\left(S_{i j}-\frac{1}{3} \frac{\partial u_{k}}{\partial x_{k}} \delta_{i j}\right)}_{\text {anisotropic }} .
$$

Of the many of ways to address the deficiencies of the Boussinesq stress-strain relation, EARSMs [20] are an effective and computationally efficient approach to express the anisotropy tensor $\boldsymbol{a}_{i j}$ as a function of a tensor basis $V_{i j}^{m}$ and scalar invariants $I_{k}$. These models add non-linear terms to the stress-strain relationship and their calculation incurs little additional computational expense. Equation (3) defines $\boldsymbol{a}_{i j}$, which is normalized by $2 \rho k$, for both the Boussinesq approximation and EARSMs

$$
\begin{aligned}
a_{i j} & \equiv \frac{\tau_{i j}}{2 \rho k}-\frac{1}{3} \boldsymbol{\delta}_{i j}, \\
a_{i j}^{B} & =-\frac{v_{t}}{k} S_{i j}^{\prime} \quad(\text { Boussinesq }), \\
a_{i j}^{E A R S M} & =-\frac{v_{t}}{k} \boldsymbol{S}_{i j}^{\prime}+f\left(V_{i j}^{1}, V_{i j}^{2}, \boldsymbol{V}_{i j}^{3}, \ldots, I_{1}, I_{2}, \ldots\right) . \\
a_{i j}^{E A R S M} & =-\frac{v_{t}}{k} \boldsymbol{S}_{i j}^{\prime}+\sum_{m=1}^{3}\left(\zeta_{m} V_{i j}^{m}\right)
\end{aligned}
$$

The tensor basis and set of scalar invariants, used to define $\boldsymbol{a}_{i j}^{E A R S M}$, are functions of the non-dimensional strain and rotation rate tensors, denoted by $s_{i j}=\tau \boldsymbol{S}_{i j}^{\prime}$ and $w_{i j}=\tau \boldsymbol{\Omega}_{i j}$; where $\tau$ is the turbulent time scale obtained from $1 / \omega$. For the flow scenario considered in this work, only the first three tensor basis functions have been considered. The selected tensor basis functions and invariants are:

$$
\begin{aligned}
& V_{i j}^{1}=s_{i j}, V_{i j}^{2}=s_{i k} w_{k j}-w_{i k} s_{k j}, \\
& V_{i j}^{3}=s_{i k} s_{k j}-\frac{1}{3} \boldsymbol{\delta}_{i j} s_{m n} s_{n m}, \\
& I_{1}=s_{m n} \boldsymbol{s}_{n m}, I_{2}=\boldsymbol{w}_{m n} w_{n m} .
\end{aligned}
$$

\section{CANONICAL TURBINE WAKE}

Motivation RANS-based turbulence models do not offer a hundred percent accurate prediction of the flow coming off the 


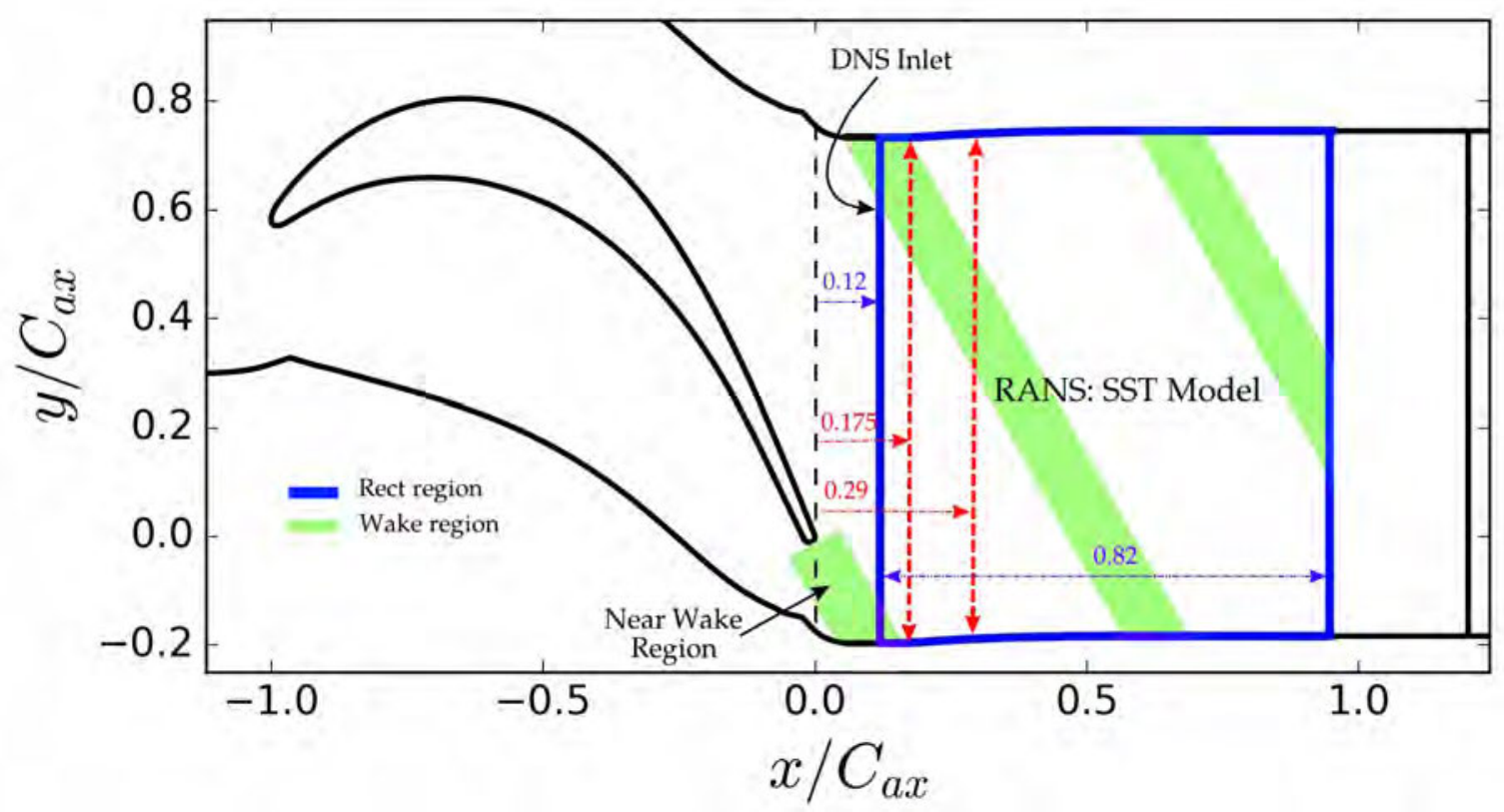

FIGURE 2: Case set up for a T106A LPT canonical wake model development.

blade into the wake region (i.e. region downstream of the blade trailing edge (TE) - shown in Fig. 2). Even if transition models are used, they cannot predict the separation characteristics accurately [12] and thus the flow coming off the blade will not be exactly the same as in high-fidelity simulations (or experiments). It has already been shown that 'CFD-driven' models do improve the wake mixing prediction in a RANS context [19,21]. Therefore, it can be argued whether 'CFD-driven' EARSMs are simply inserting another error in order to mitigate the erroneous flow coming from the blade TE into the wake region, or whether they are capturing the right physics in order to improve flow prediction? It is best to therefore assess the performance of the 'CFD-driven' models on a canonical turbine wake case first, which have correct inflow conditions based on high-fidelity data. To achieve this, RANS calculations and 'CFD-driven' EARSM development was conducted for a pitch-wise periodic 2D box-like region (Rect region in Fig. 2) by prescribing experimentally-validated DNS [22] profiles at the inlet of this domain, which ensures that the inflow conditions are correct. This will inform us whether the 'CFD-driven' EARSMs can improve the flow prediction in cases with the correct inflow conditions and are not introducing additional errors that might cancel others.

Case Setup \& Model Development The domain for this set of calculations is shown in Fig. 2. Note that the origin is chosen such that it coincides with the T106A LPT blade TE. A rectangular box (Rect-region) which commences $12 \% \mathrm{C}_{a x}$ downstream of the $\mathrm{TE}$ (which excludes the near-wake region) with a length of $0.82 \mathrm{C}_{a x}$ is chosen as the domain region. It is known quite well that RANS cannot capture the flow in the near-wake region properly [12] due to the high deterministic unsteadiness, and this will be quantified later in this work. The near-wake region is thus excluded as a first step. The length of the domain was chosen after domain convergence studies, to ensure that a consistent rate of decay of mean flow quantities was met. At $12 \% \mathrm{C}_{a x}$ downstream from the TE, time-averaged stagnation pressure, stagnation temperature, flow angle, TKE and $\omega$ ( $\omega$ was derived from a 'frozen' calculation using the $k \omega$-SST model
- refer to [12] regarding details of 'frozen' calculations) profiles from an LPT DNS at $\operatorname{Re}_{2 i s}=100,000$ [22] were prescribed. At the outlet the static pressure based on the DNS data was prescribed. All other variables were set to a zero gradient boundary condition at the outlet. First, a baseline case was run using the $k \omega$-SST model with the code TRAF [23]. No transition model was selected as the flow downstream of the TE is purely turbulent. After the baseline case was executed, an EARSM was developed for the wake region of the rectangular box using the 'CFD-driven' approach. The cost function evaluation is located at $17.5 \% \mathrm{C}_{a x}(15 \% \mathrm{C})$ and $29 \% \mathrm{C}_{a x}(25 \% \mathrm{C})$ downstream of the blade TE. The equation of the resulting EARSM for the canonical wake (CW model) is

$$
\begin{aligned}
a_{i j}^{E A R S M-C W} & =-\frac{v_{t}}{k} S_{i j}^{\prime}+\left(-0.76+I_{1}\right) V_{i j}^{1}+\left(-2.6+0.6 I_{1}\right) V_{i j}^{2} \\
& +\left(1+0.86 I_{1}+I_{2}+I_{2}^{2}\right) V_{i j}^{3} .
\end{aligned}
$$

Flow Characteristics This section discusses the mean flow characteristics of the baseline (using the Boussinesq approximation), trained (Eq. 5) and DNS cases. The results from the trained model have Eq. 5 implemented only in the wake region and in between these regions, the Boussinesq approximation is used. In realtime applications, the wake region can be determined by certain TKE thresholds. A user may also chose to use a ramping function when transitioning between different stress terms in the different regions. It was found, however, that using a ramping function did not perceivably change the flow field for the cases presented in this work. No numerical instabilities were reported either when a ramping function was not used. Figure 3 shows the evolution of the peak wake loss across the entire domain. At the inlet, the baseline and trained models match the DNS, as DNS profiles are being prescribed. However, when going further downstream the peak wake loss from the baseline case starts to deviate from the DNS, until it starts to maintain a constant difference with the DNS peak wake loss from $40 \% \mathrm{C}_{a x}$ onwards. The 'CFD-driven' EARSM, on the other hand, follows the peak wake loss evolution right from the 


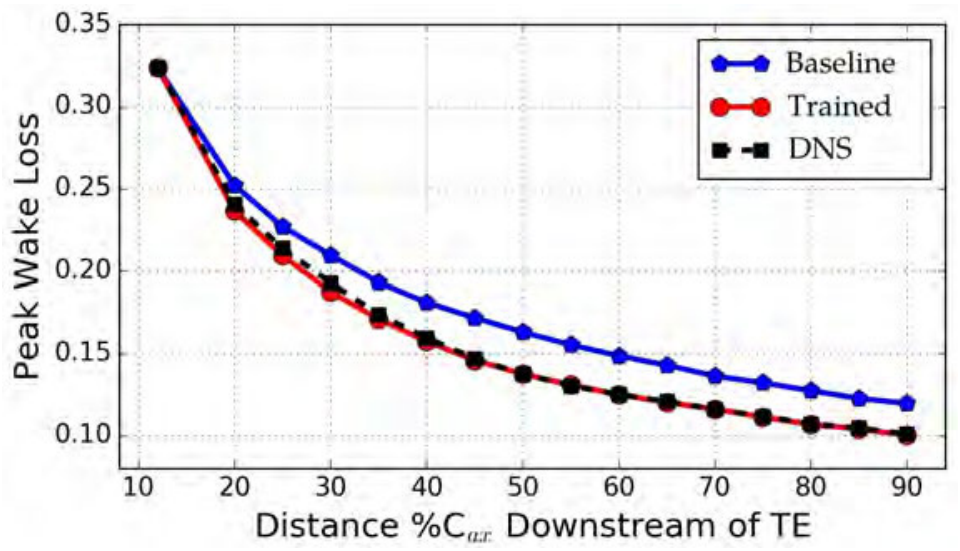

FIGURE 3: Variation of peak wake loss with distance downstream of the TE.

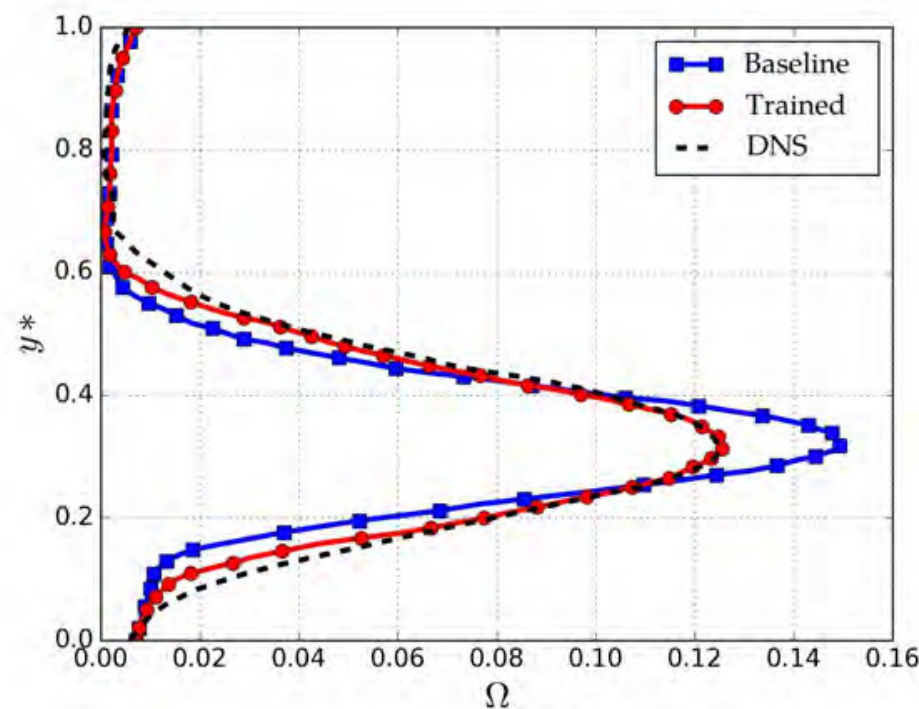

FIGURE 4: Wake loss profile $60 \% \mathrm{C}_{a x}$ downstream of the TE.

beginning of the domain to the end. This gives us tremendous confidence in the ability of the 'CFD-driven' models to find the right model form and coefficients such that it can match the DNS peak wake loss.

Wake loss profiles are shown at $60 \% \mathrm{C}_{a x}$ downstream of the TE in Fig. 4. The trained model not only gets the right peak wake loss but also closely matches the spreading rate of the DNS wake loss profile. The trained model, however, suffers slightly in the wake-periphery region $\left(y^{*} \approx 0.2 \& y^{*} \approx 0.5\right)$, which are also the junctions where the trained models are switched on and off, respectively. This can be attributed to RANS not being able to mix out the flow in this region properly. Nevertheless, the wake loss profiles in Fig. 4 show that the RANS-based 'CFD-driven' approach is able to provide models that can accurately capture the wake mixing when the right inflow conditions are provided. It can thus be confidently stated that the 'CFD-driven' models improve the wake mixing prediction by capturing correct trends in flow physics, and not by introducing additional errors to cancel potential other errors.

The variation of integral loss in the region from $12 \% \mathrm{C}_{a x}$ to $90 \% \mathrm{C}_{a x}$ is shown in Fig. 5. At the inlet the baseline and trained

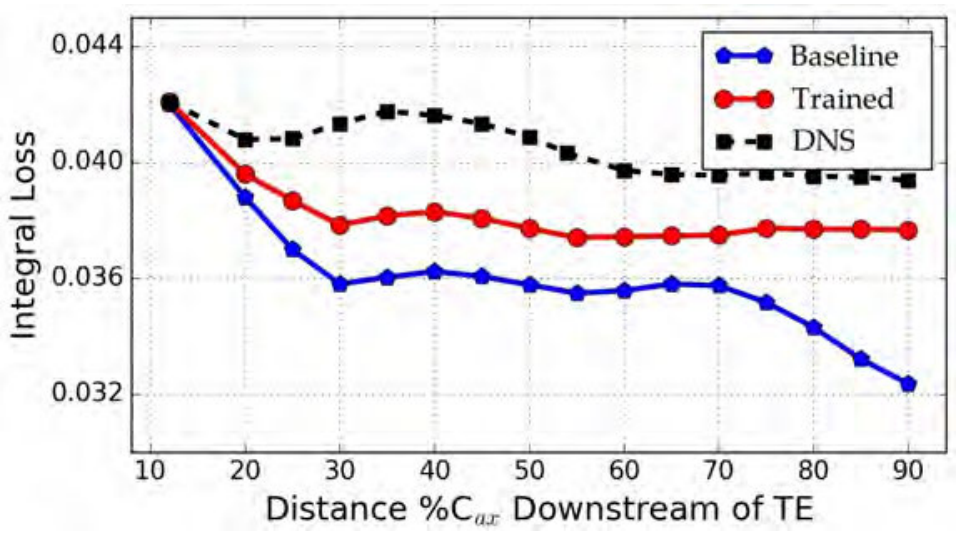

FIGURE 5: Variation of integral loss with distance downstream of the TE.

cases match the integral loss of the DNS. However, as the flow develops, the integral loss slowly starts to shift away from the DNS, more so in the baseline case. Even the trained model is not able to match the integral loss. This is due to the shortcoming of RANS not being able to predict the wake loss profiles accurately in the wake-periphery region. This portion of the wake loss profile accounts for the deviation in the trained case with respect to the DNS. However, the trained case matches the overall trend of the integral loss better than the baseline case, especially after $70 \% \mathrm{C}_{a x}$. Beyond $60 \% \mathrm{C}_{a x}$ the integral loss from the trained model shows an error of only about $5 \%$ with respect to the integral loss from the DNS.

As a summary, when the correct inflow conditions from highfidelity data were prescribed, the 'CFD-driven' approach had the ability to closely match the DNS wake evolution, which is a very promising result for RANS-based turbulence model development. It can therefore be assumed with increased confidence that 'CFDdriven' models capture the right trends in physics to improve the overall wake mixing for full-blade scenarios. This will be assessed in the following section.

\section{RANS FOR FULL-BLADE CASES}

This section aims to extend the analysis conducted for the LPT canonical wake to the full LPT blade cases at two Reynolds numbers, $\operatorname{Re}_{2 i s}=60,000$ and $\operatorname{Re}_{2 i s}=100,000$, with an inlet free-stream turbulence intensity of $4 \%$ (i.e. steady inflow conditions) and isentropic exit Mach number of 0.4 . The $\gamma-\operatorname{Re}_{\theta}$ turbulence model has been used as there is laminar separation in the boundary layer of the suction side. EARSMs were developed for each of the Reynolds numbers using profiles at different locations in the wake for the cost function (Eq. 1) to evaluate the impact of training location on model performance.

Training Regions Selecting the right training region (or the region where the EARSM will be applied) in the wake area, as part of the training process, is of utmost importance in order to get the best quality model for the 'CFD-driven' approach. Weatheritt et al. [14] suggested that the near-wake region affects the nature of the trained model for turbomachinery cases. The study in this section analyses what portion of the near-wake region can be included in the training region (i.e. the 'CFD-driven' models are switched on after excluding a certain portion of the near-wake region), without causing the 'CFD-driven' approach to return models that perform worse than the Boussinesq approximation. 

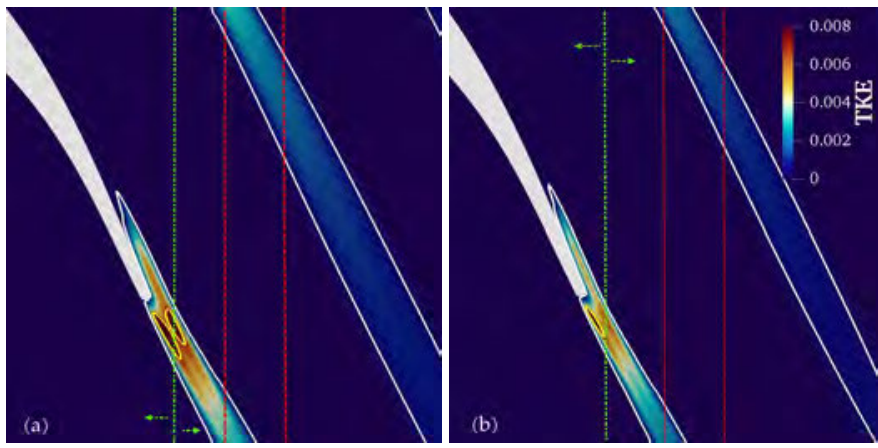

FIGURE 6: TKE contours for (a) $\operatorname{Re}_{2 i s}=60,000$ and (b) $\mathrm{Re}_{2 i s}=100,000$ from RANS calculations in the TE and wake regions of an LPT blade. Additional white and yellow contour lines at $\mathrm{TKE}=0.0004$ and $\mathrm{TKE}=0.007$, respectively, are shown. The red lines denote the cost function evaluation locations $\left(17.5 \% \mathrm{C}_{a x}\right.$ and $29 \% \mathrm{C}_{a x}$ downstream of the blade TE). A green line has been drawn at (a) $6 \% \mathrm{C}_{a x}$ and (b) $4 \% \mathrm{C}_{a x}$ downstream of the $\mathrm{TE}$, which denotes the onset of the training region. The area to the right of the green line and between the white contours is where the proposed EARSMs are applied.

Figure 6 shows the training regions on a TKE contour plot generated via RANS using the Boussinesq approximation throughout the entire flow domain at (a) $\operatorname{Re}_{2 i s}=60,000$ and (b) $\operatorname{Re}_{2 i s}=100,000$. Additional TKE contours are highlighted, at TKE $=0.0004$ (white) and TKE $=0.007$ (yellow). The red lines denote the cost function (Eq. 1) evaluation locations $\left(17.5 \% \mathrm{C}_{a x}\right.$ and $29 \% \mathrm{C}_{a x}-$ which are the same locations of the cost function evaluation as in the canonical turbine wake case). A green line has been drawn at (a) $6 \% \mathrm{C}_{a x}$ and (b) $4 \% \mathrm{C}_{a x}$ downstream of the TE, which denotes the onset of the training region. The region used for training is the one which intersects the area to the right of the green line and the area between the white contours (i.e. wake area). Outside of the training area, the Boussinesq approximation is applied. To analyse the effect of the near-wake portion on the cost function and the models developed, the green line is moved to the left and right (as shown by the green arrows), i.e. between $0 \% \mathrm{C}_{a x}$ (at blade TE) to $12 \% \mathrm{C}_{a x}$ downstream of the blade TE.

Observations \& Analysis Figure 7 primarily shows the effect of the training region and Reynolds numbers on the value of the cost functions $\left(J_{60 k}^{C F D}\right.$ and $J_{100 k}^{C F D}-$ i.e. the cost functions from Eq. 1 at $\operatorname{Re}_{2 i s}=60,000$ and $\operatorname{Re}_{2 i s}=100,000$ ) which are used for evaluation in the 'CFD-driven' approach. The $x$-axis shows the starting point of the training region as a function of percentage axial chord downstream of the blade TE (i.e. green line in Fig. 6). In Fig. 7 the cost functions $J_{60 k}^{C F D}$ and $J_{100 k}^{C F D}$ are evaluated with the Boussinesq approximation (baseline case) being used throughout the entire flow domain. The resulting value of the cost function, which is obviously independent of the training region, is plotted by a red line in both plots in Fig. 7. The closer the value of the cost function is to zero, the closer is the wake loss profile to the reference DNS data.

Figure 7(a) shows the value of $J_{60 k}^{C F D}$ from 18 RANS calculations. Note that hundreds of RANS calculations were already conducted as part of the model development process to find the best model for a particular training region. Only the results from the best model for a particular training region are shown in Fig. 7. Six of these calculations are from models that have been developed on the

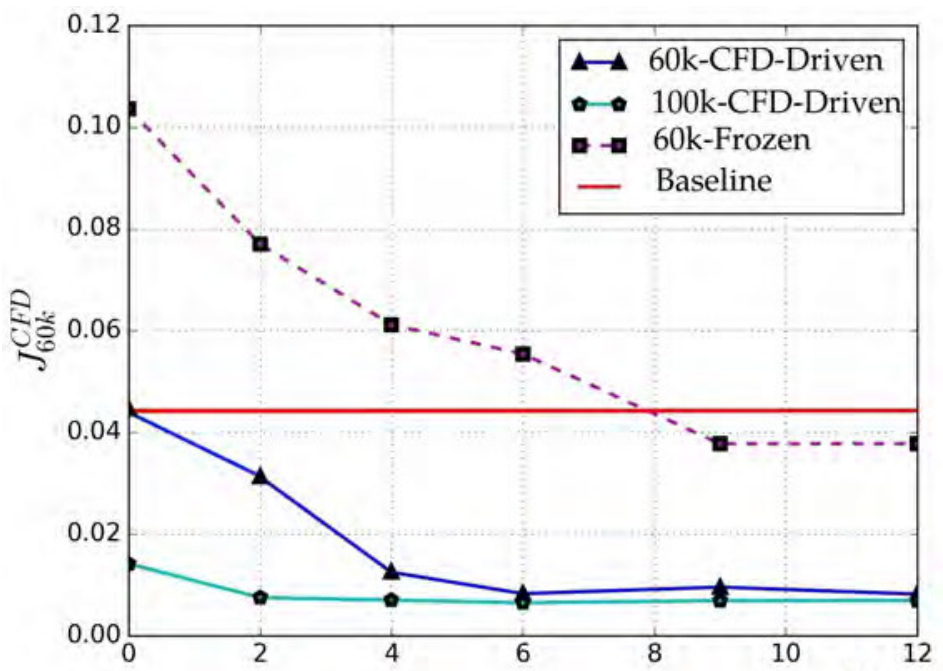

(a)

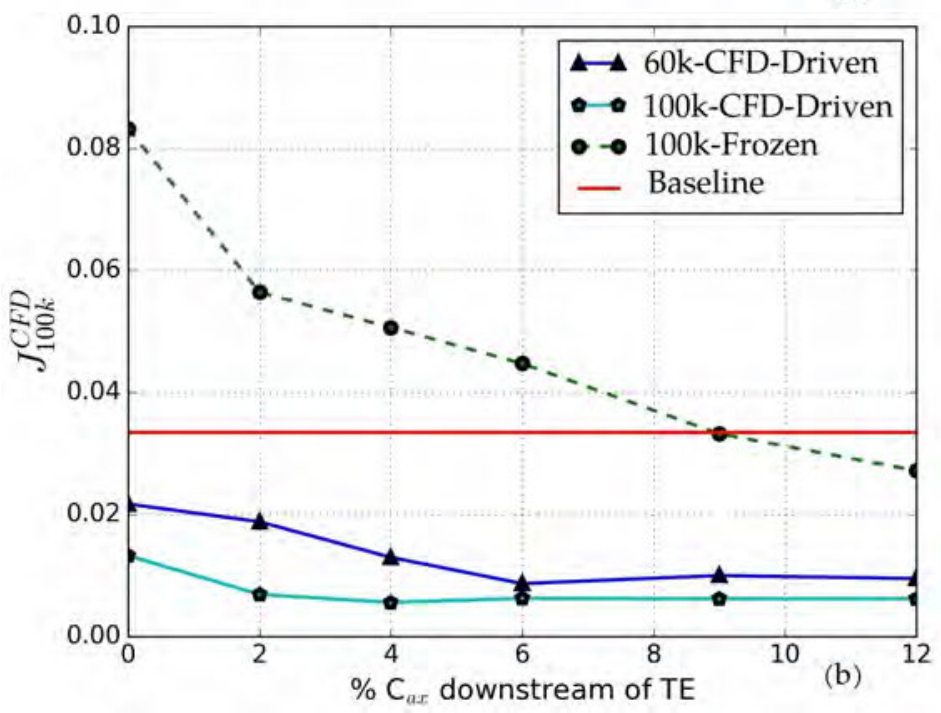

FIGURE 7: Influence of training region downstream of blade TE on the 'CFD-driven' cost function (Eq. 1) at (a) $\operatorname{Re}_{2 i s}=60,000$ and (b) $\operatorname{Re}_{2 i s}=100,000$.

$\mathrm{Re}_{2 i s}=60,000$ case for the respective training region with the 'CFDdriven' approach (60k-CFD-Driven). Six of these calculations are from models that have been trained on the $\operatorname{Re}_{2 i s}=100,000$ case via the 'CFD-driven' approach (100k-CFD-Driven) and tested on the corresponding region at $\operatorname{Re}_{2 i s}=60,000$. The remaining six calculations are from models that have been developed using the 'frozen' approach (see Eq. 7) at $\operatorname{Re}_{2 i s}=60,000$ (60k-Frozen). The performance of these models is then tested against $J_{60 k}^{C F D}$ in order to assess the wake loss or mean flow development. Figure 7(b) shows the value of $J_{100 k}^{C F D}$ from 18 similar RANS calculations as in Fig. 7(a) at $\operatorname{Re}_{2 i s}=100,000$. It should be noted that in Fig. 7, models developed on a particular training region have been tested only on the corresponding training region that they were developed on, even if they are tested across different Reynolds numbers.

With the 60k-CFD-Driven models tested at $\operatorname{Re}_{2 i s}=60,000$ (Fig. $7(\mathrm{a})$ ), if a training region is considered right from downstream of the blade TE, the cost function returned is the same as the baseline case, as the 'CFD-driven' approach is unable to find a better solution than the Boussinesq approximation. The models begin to show convergence in the value of $J_{60 k}^{C F D}$ around $5-6 \% \mathrm{C}_{a x}$ downstream of 
the blade TE. Beyond $6 \% \mathrm{C}_{a x}$ downstream of the blade TE, these models show an improvement of about four times in the value of $J_{60 k}^{C F D}$ over the baseline case. When the 100k-CFD-Driven models are tested at $\operatorname{Re}_{2 i s}=100,000$, they perform much better than the baseline case right from the blade TE and they begin to show convergence in the value of $J_{100 k}^{C F D}$ around $3-4 \% \mathrm{C}_{a x}$ downstream of the blade TE. Beyond this point they show an improvement of more than four times over the baseline case. This shows that beyond a certain point in both the cases, there are significant improvements in the wake loss prediction over the baseline case.

Figure 6 shows a TKE contour line at TKE $=0.007$, which is part of the shear layer near the blade TE in both the cases. The shear layer features higher TKE levels at $\operatorname{Re}_{2 i s}=60,000$ than at $\operatorname{Re}_{2 i s}=100,000$, which is largely due to the higher inherent deterministic unsteadiness caused by more pronounced vortex shedding due to an open separation. If a training region is considered beyond most of the shear layer with high TKE magnitude (i.e. strong shear layer), the cost function value begins to converge. The portion to the right of the green line at $6 \% \mathrm{C}_{a x}$ downstream of the TE in Fig. 6 (a) excludes most of the strong shear layer and hence the value of the cost function begins to converge around $6 \% \mathrm{C}_{a x}$ downstream of the TE. At $\operatorname{Re}_{2 i s}=100,000$ the shear layer is much weaker than at $\mathrm{Re}_{2 i s}=60,000$ due to a closed separation and this leads to less coherent flow. At $\operatorname{Re}_{2 i s}=100,000$, the green line at $4 \% \mathrm{C}_{a x}$ downstream of the TE shown in Fig. 6, is beyond the strong shear layer and thus the value of the cost function converges around 3-4\% $\mathrm{C}_{a x}$ downstream of the TE. The 'CFD-driven' approach at $\mathrm{Re}_{2 i s}=60,000$ is unable to find a solution which is performing better than the baseline case if the training region begins from the blade $\mathrm{TE}$ (i.e. $0 \% \mathrm{C}_{a x}$ downstream of the blade TE) due to the high deterministic unsteadiness in this area. This suggests that for cases with an open separation, the near-wake region propagates additional errors into the flow downstream of the TE, which cannot be accounted for by the RANSbased 'CFD-driven' approach effectively.

In order to assess the robustness of the models developed, the 60k-CFD-driven and 100k-CFD-driven models were each tested at the Reynolds number other than they were developed on, while keeping the model execution region similar to the training region. An interesting point to note is that the 100k-CFD-Driven models perform much better than the 60k-CFD-Driven models in the nearwake regions at both Reynolds numbers. This is attributed to the fact that the 100k-CFD-Driven models have to encounter lower deterministic unsteadiness while training, a phenomenon which steady RANS is unable to capture. If a training region starting from beyond $6 \% \mathrm{C}_{a x}$ downstream of the blade TE is considered, both the 60k-CFD-Driven and 100k-CFD-Driven models perform comparably well on both cases. In the near-wake region, it has been found that there is high pressure-velocity coupling; whose effect is modeled by a term which appears in the TKE transport equation [1]. This region thus is difficult to model just by improving the accuracy of the anisotropy tensor (EARSMs). Since the 'CFD-driven' approach focuses on improving the mean flow prediction by modifying the anisotropy tensor, the near-wake region has to be excluded for model implementation purposes. In essence, the greater the deterministic unsteadiness in the flow, the greater the portion of the wake near the TE that has to be excluded from the training region in order to obtain a model that performs well in the far-wake. It is anticipated that URANS should be able to resolve the deterministic unsteadiness in the near-wake region and that is the subject of the next section (i.e. Unsteady RANS for full-blade cases).
By scrutinizing the different models obtained from the 'CFDdriven' training, we find that in the current case the term that plays the most important role in improving the wake mixing prediction in the RANS context is $\boldsymbol{V}_{i j}^{1}$. The coefficient of this term governs the net additional turbulent diffusion that is required in order to enhance the wake mixing prediction as compared to the Boussinesq approximation. For the canonical turbine wake case ( $\mathrm{CW}$ model in Eq. 5), this coefficient is approximately -0.76 (note the constant term dominates out of all terms in the coefficient of the respective tensor basis functions). For the corresponding full-blade case (i.e. at $\operatorname{Re}_{2 i s}=100,000$ ), when the near-wake region portion is excluded up to $4 \% \mathrm{C}_{a x}$ and beyond (or the training region commences from $4 \% \mathrm{C}_{a x}$ and beyond) the coefficient of the $\boldsymbol{V}_{i j}^{1}$ term is -2 (note that the model has the same functional form whether the training region commences at $4 \% \mathrm{C}_{a x}$ or at $12 \% \mathrm{C}_{a x}$ - which is visible by the value of $J_{100 k}^{C F D}$ in Fig. 7(b)). The model for the full-blade (FB model) case is shown below

$$
a_{i j}^{E A R S M-F B}=-\frac{v_{t}}{k} \boldsymbol{S}_{i j}^{\prime}+(-2) \boldsymbol{V}_{i j}^{1}+\left(-2+I_{2}\right) V_{i j}^{2}+\left(-I_{2}\right) V_{i j}^{3} .
$$

This shows us that the coefficient derived from the canonical turbine wake model, which depicts the right development of the wake mixing, is smaller compared to the full-blade model, which overcompensates for the near-wake region that is dominated by the strong vortex shedding.

For comparison, an assessment of the performance of the anisotropy-based 'frozen' approach in the near-wake region in the context of steady RANS has been conducted. The models were developed using the cost function for the 'frozen' approach (Eq. 7) which aims to minimize the sum of the difference between the individual components of the anisotropy tensors, i.e. the anisotropy tensor that is created from the EARSMs $\left(a_{i j}^{E A R S M}\right)$ and the one from the high-fidelity flow field $\left(a_{i j}^{H i-F i}\right)$, as

$$
J\left(a_{i j}^{E A R S M}\right)=\frac{1}{M} \sum_{n=1}^{M} \sum_{i=1}^{3} \sum_{j \leq i}\left(\left|a_{i j}^{H i-F i}-a_{i j}^{E A R S M}\right|\right)^{2}
$$

where $M$ is the total number of points in the training region. The models were then were run as RANS calculations and then evaluated in terms of the cost functions in Eq. 1. At both Reynolds numbers the models developed via the 'frozen' approach perform better than the baseline case only beyond $8-9 \% \mathrm{C}_{a x}$ downstream of the blade TE. Due to the high magnitude of anisotropy in the wake region near the blade TE as compared to the rest of the wake, the 'frozen' approach is unable provide a model which can predict the mean flow accurately at a downstream location.

To summarize, the models based on the 'CFD-driven' cost functions offer significantly better performance over the models developed via the 'frozen' approach in terms of the wake loss, as they are able to capture certain flow-history effects which the 'frozen' approach is unable to perceivably capture. As the 'CFD-driven' models are able to better capture history effects of the flow, the portion of the near-wake region to be excluded for developing a model that performs better than the baseline case, is smaller than the 'frozen' approach. 

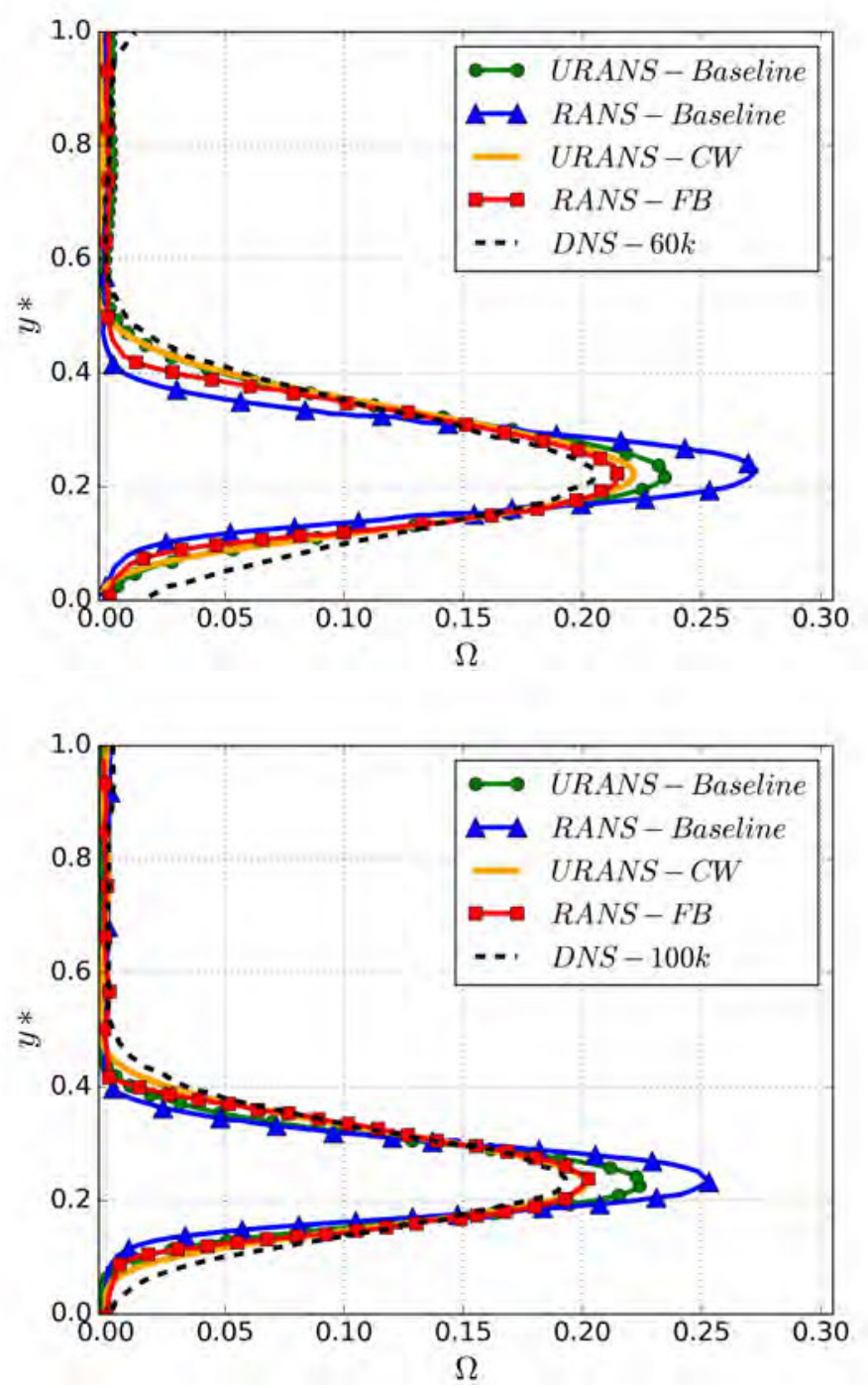

FIGURE 8: Wake loss profiles at $25 \% \mathrm{C}$ downstream of the blade TE at (a) $\operatorname{Re}_{2 i s}=60,000$ and (b) $\operatorname{Re}_{2 i s}=100,000$, for the baseline RANS and URANS cases, the FB model in RANS and CW model in URANS.

\section{UNSTEADY RANS FOR FULL-BLADE CASES}

From the previous section it is quite clear that RANS cannot capture the near-wake region properly. When the near-wake region is excluded, GEP is able to produce a model that predicts the wake mixing accurately. RANS is unable to capture the deterministic unsteadiness close to the blade and hence it does not perform well in that region. By switching to URANS it is expected that the nearwake region will be captured better and the wake mixing prediction will be improved over steady RANS. In this section the performance of URANS is assessed for cases at both Reynolds numbers.

Wake Loss Profiles Figure 8 shows wake loss profiles located $25 \% \mathrm{C}$ downstream of the blade $\mathrm{TE}$ for $\mathrm{Re}_{2 i s}=60,000$ and $\operatorname{Re}_{2 i s}=100,000$. The baseline cases for RANS and URANS use the Boussinesq approximation throughout the entire domain. At both Reynolds numbers it is visible that the URANS baseline case is performing better than the baseline RANS case as URANS can better capture the deterministic unsteadiness in the near-wake region. However, there is still a lack of accuracy compared to DNS data.
Since the near-wake region is captured better with URANS, it was decided to test the model developed on the canonical turbine wake (CW model - Eq. 5), which is representative of the wake mixing phenomena when the near-wake region is captured quite well. For comparison, we also show the best performing trained model in the steady RANS context, using the FB model - i.e. Eq. 6. The calculations with trained models have the respective EARSMs applied in the region starting from about $5 \% \mathrm{C}_{a x}$ downstream of the blade TE and encompassed by the contour TKE=0.0004 (see Fig. 6).

At both Reynolds numbers, the model developed on the canonical turbine wake is able to perform significantly better than the baseline URANS calculation - both in terms of wake width and peak wake loss prediction. Thus we find that the non-linear closure producing the best results depends on whether one wants to perform steady or unsteady RANS. If steady RANS are to be conducted, the CFD-driven approach applied to a full-blade data set produces a model that adds a larger amount of diffusion to account for the inability of steady RANS to capture the near-wake. If unsteady RANS are to be used that are able to resolve the near-wake vortex shedding, using a model trained on the far wake, or here the canonical wake case, will provide the correct diffusion to maintain an accurate wake development throughout. The analysis here supports the findings of Lav et al. [17], who showed that machine-learnt EARSMs implemented in RANS calculations have larger values of additional diffusion coefficients than those required for URANS calculations. It should also be noted that both the CW and FB models were developed at $R e_{2 i s}=100,000$, which has a wake which originates from a boundary layer with closed separation. Both of these models perform well at $R e_{2 i s}=60,000$, which has a wake originating from an open separation in the boundary layer. This shows that the models developed are robust across topologically different flows. From the above analysis the benefits of the 'CFD-driven' model development approach become clear and it is shown that this approach is able to create physically correct EARSMs to augment the wake mixing prediction.

\section{CONCLUSIONS}

This study explored the integration of GEP with RANS based CFD for the development of EARSMs to increase the accuracy of wake mixing prediction of LPTs. With the 'CFD-driven' approach, the user is given the freedom to define a cost function based on a mean flow or secondary statistic of their choice, unlike the 'frozen' approach which needs a cost function based on a secondary statistic which is present in the stress-strain closure term. Due to the integration of CFD as part of the model development process, the resultant 'CFD-driven' models capture flow history effects better than their 'frozen' counterparts.

In order to ensure that the models from the 'CFD-driven' approach are actually improving the accuracy of the wake mixing prediction and not just inserting another error to correct the original error from a RANS calculation, 'CFD-driven' model development was conducted for a canonical turbine wake by prescribing inlet profiles from a well-validated DNS. When the correct inflow conditions from DNS data were prescribed, the 'CFD-driven' approach had the ability to closely match the DNS wake development and maintain an accurate peak wake loss throughout the domain. Extending this analysis to full-blade cases, it was found that the functional form of the 'CFD-driven' model is sensitive to the training region on account of the presence of deterministic unsteadiness in 
the near-wake region. This study quantified the amount of the nearwake region that has to be excluded for developing a model that performs well in the far-wake at $R e_{2 i s}=60,000$ and $R e_{2 i s}=100,000$. It was found that models developed on the full-blade cases have an additional diffusion coefficient (coefficient of $V_{i j}^{1}$ ) which is nearly 2.5 times larger in magnitude than in the canonical turbine wake case. This is because the full-blade cases over-compensates for the near-wake region, whereas in the canonical turbine wake case, a smaller diffusion coefficient can successfully predict the wake development because the incoming flow is correct. This issue can be remedied by using physically consistent models (i.e. the canonical wake model) in URANS calculations as URANS is able to model the near-wake region better than steady RANS due to its ability to capture deterministic unsteadiness.

While the models trained in the present study are limited to wake mixing, the 'CFD-driven' machine learning is shown to be a promising framework for general turbulence model development and can be extended to improve the prediction of other flow features as well.

\section{ACKNOWLEDGMENTS}

This work was supported by resources provided by the Pawsey Supercomputing Centre using the Magnus supercomputer, with funding from the Australian Government and the Government of Western Australia. The support by the Australian Government Research Training Program Scholarship is acknowledged.

\section{NOMENCLATURE}

$\boldsymbol{a}_{i j} \quad$ Normalized anisotropy tensor

$B \quad$ Boussinesq

C Blade chord length

$I_{k} \quad$ Scalar invariant

$J \quad$ Cost function for the 'frozen' approach

$J^{C F D}$ Cost function for the 'CFD-driven' approach

$k \quad$ Turbulent kinetic energy

$M \quad$ Total points in training region for the 'frozen' approach

$P \quad$ Pressure

Re Reynolds number

$S_{i j} \quad$ Strain rate: $\frac{1}{2}\left(\partial_{x_{j}} U_{i}+\partial_{x_{i}} U_{j}\right)$

$\boldsymbol{S}_{i j}^{\prime} \quad$ Deviatoric component of strain rate: $\boldsymbol{S}_{i j}-\frac{1}{3} \boldsymbol{\delta}_{i j} S_{k k}$

$V_{i j}^{m} \quad$ Tensor basis function

$w \quad$ Pitch

$x, y \quad$ Cartesian coordinates

$y^{*} \quad$ Non-dimensional pitchwise coordinate

$\gamma \quad$ Intermittency

$\boldsymbol{\delta}_{i j} \quad$ Kronecker Delta: $\delta_{i j}=0$ if $i \neq j, \delta_{i j}=1$ if $i=j$

$\zeta_{m} \quad$ Coefficient of $V_{i j}^{m}$

$\mu_{t} \quad$ Eddy viscosity

$\rho \quad$ Density

$\tau \quad$ Turbulent time scale: $(1 / \omega)$

$\tau_{i j} \quad$ Reynolds stress tensor: $\rho \overline{u_{i}^{\prime} u_{j}^{\prime}}$

$\omega$ Specific dissipation rate

$\Omega \quad$ Loss coefficient

$\boldsymbol{\Omega}_{i j} \quad$ Rotation rate tensor: $\frac{1}{2}\left(\partial_{x_{j}} U_{i}-\partial_{x_{i}} U_{j}\right)$

\section{SUBSCRIPTS / SUPERSCRIPTS}

\section{Inlet}

2

$60 k$ Reynolds number of 60,000

$100 k$ Reynolds number of 100,000

ax Axial

is Isentropic

$t \quad$ Stagnation quantity

$\theta \quad$ Momentum thickness

\section{ACRONYMS}

CFD Computational fluid dynamics

CW Canonical wake

DNS Direct numerical simulation

EARSM Explicit algebraic Reynolds stress model

FB Full-blade

GEP Gene expression programming

Hi-Fi High-fidelity

LES Large eddy simulation

LPT Low pressure turbine

RANS Reynolds averaged Navier-Stokes

SST Shear stress transport

TE Trailing edge

TKE Turbulent kinetic energy

URANS Unsteady RANS

\section{REFERENCES}

[1] Leschziner, M., 2015. Statistical Turbulence Modelling for Fluid Dynamics - Demystified: an Introductory Text for Graduate Engineering Students. World Scientific, Oxford, UK.

[2] Medic, G., 2019. "Impact of Vision 2030 on CFD Practices in Propulsion Industry”. In AIAA Aviat. 2019 Forum, p. 2943.

[3] Singh, A. P., Medida, S., and Duraisamy, K., 2017. "MachineLearning-Augmented Predictive Modeling of Turbulent Separated Flows over Airfoils". AIAA J., 55(7), pp. 2215-2227.

[4] Wang, J.-X., Wu, J.-L., and Xiao, H., 2017. "A Physics Informed Machine Learning Approach for Reconstructing Reynolds Stress Modeling Discrepancies Based on DNS Data". Phys. Rev. Fluids, 2(3), p. 034603.

[5] Wu, J.-L., Xiao, H., and Paterson, E., 2018. "PhysicsInformed Machine Learning Approach for Augmenting Turbulence Models: A Comprehensive Framework". Phys. Rev. Fluids, 3(July), p. 074602.

[6] Ling, J., Templeton, J., and Kurzawski, A., 2016. "Reynolds Averaged Turbulence Modelling Using Deep Neural Networks with Embedded Invariance". J. Fluid Mech, 807(2016), pp. 155-166.

[7] Milani, P. M., Ling, J., and Eaton, J. K., 2019. "Physical Interpretation of Machine Learning Models Applied to Film Cooling Flows". J. Turbomach., 141(1), p. 011004.

[8] Duraisamy, K., Iaccarino, G., and Xiao, H., 2019. "Turbulence Modeling in the Age of Data”. Annu. Rev. Fluid Mech., 53(1), pp. 357-377.

[9] Ferreira, C., 2001. "Gene Expression Programming: a New Adaptive Algorithm for Solving Problems". Complex Syst., 13(2), pp. 87-129.

[10] Weatheritt, J., and Sandberg, R. D., 2016. "A Novel Evolutionary Algorithm Applied to Algebraic Modifications of the RANS Stress-Strain Relationship". J. Comput. Phys., 325, pp. 22-37.

[11] Akolekar, H. D., Sandberg, R. D., Hutchins, N., Michelassi, V., and Laskowski, G., 2019. "Machine-Learnt Turbulence 
Closures for Low Pressure Turbines with Unsteady Inflow Conditions". J. Turbomach., 141(10), p. 101009.

[12] Akolekar, H. D., Weatheritt, J., Hutchins, N., Sandberg, R. D., Laskowski, G., and Michelassi, V., 2019. "Development and Use of Machine-Learnt Algebraic Reynolds Stress Models for Enhanced Prediction of Wake Mixing in Low-Pressure Turbines". J. Turbomach., 141(4), pp. 041010:1-11.

[13] Wu, J., Xiao, H., Sun, R., and Wang, Q., 2019. "Reynoldsaveraged NavierStokes equations with explicit data-driven Reynolds stress closure can be ill-conditioned". J. Fluid Mech., 869, pp. 553-586.

[14] Weatheritt, J., Pichler, R., Sandberg, R. D., Laskowski, G., and Michelassi, V., 2017. "Machine Learning for Turbulence Model Development Using a High Fidelity HPT Cascade Simulation". In ASME Turbo Expo 2017 Turbomach. Tech. Conf. Expo., pp. GT2017-63497.

[15] Sandberg, R. D., Tan, R., Weatheritt, J., Ooi, A., Haghiri, A., Michelassi, V., and Laskowski, G., 2018. "Applying Machine Learnt Explicit Algebraic Stress and Scalar Flux Models To a Fundamental Trailing Edge Slot". J. Turbomach., 140(10), p. 101008.

[16] Weatheritt, J., Zhao, Y., Sandberg, R. D., Mizukami, S., and Tanimoto, K., 2019. "Data-driven scalar-flux model development with application to jet in cross flow". Int. J. Heat Mass Transf., p. 118931.

[17] Lav, C., Sandberg, R. D., and Philip, J., 2019. "A framework to develop data-driven turbulence models for flows with organised unsteadiness". J. Comput. Phys., 383, pp. 148-165.

[18] Schoepplein, M., Weatheritt, J., Sandberg, R., Talei, M., and Klein, M., 2018. "Application of an evolutionary algorithm to LES modelling of turbulent transport in premixed flames". $J$. Comput. Phys., 374, pp. 1166-1179.

[19] Zhao, Y., Akolekar, H. D., Weatheritt, J., Michelassi, V., and Sandberg, R. D., 2020. "Turbulence Model Development using CFD-Driven Machine Learning". J. Comput. Phys., under rev.

[20] Pope, S. B., 1975. "A More General Effective-Viscosity Hypothesis". J. Fluid Mech., 72(2), pp. 331-340.

[21] Akolekar, H. D., Zhao, Y., Sandberg, R. D., Hutchins, N., and Michelassi, V., 2019. "Turbulence Model Development for Low \& High Pressure Turbines Using a Machine Learning Approach". In Int. Soc. Air Breath. Engines.

[22] Michelassi, V., Chen, L. W., Pichler, R., and Sandberg, R. D., 2015. "Compressible Direct Numerical Simulation of Low Pressure Turbine - Part II: Effect of Inflow Disturbances". J. Turbomach., 137(7), p. 71005.

[23] Arnone, A., Liou, M. S., and Povinelli, L. A., 1992. "NavierStokes Solution of Transonic Cascade Flows Using". $J$. Propuls. Power, 8(2), pp. 410-417. 


\section{University Library}

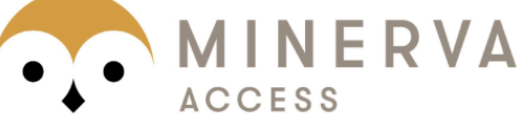

A gateway to Melbourne's research publications

Minerva Access is the Institutional Repository of The University of Melbourne

Author/s:

Akoleka, HD;Zhao, Y;Sandberg, R;Pacciani, R

Title:

INTEGRATION OF MACHINE LEARNING AND COMPUTATIONAL FLUID DYNAMICS TO DEVELOP TURBULENCE MODELS FOR IMPROVED TURBINE WAKE MIXING PREDICTION

Date:

2021

Citation:

Akoleka, H. D., Zhao, Y., Sandberg, R. \& Pacciani, R. (2021). INTEGRATION OF MACHINE LEARNING AND COMPUTATIONAL FLUID DYNAMICS TO DEVELOP TURBULENCE MODELS FOR IMPROVED TURBINE WAKE MIXING PREDICTION. Proceedings of ASME Turbo Expo 2020: Turbomachinery Technical Conference and Exposition GT2020, 2C-2020, Search Results Web results ASME: The American Society of Mechanical Engineers. https:// doi.org/10.1115/GT2020-14732.

Persistent Link:

http://hdl.handle.net/11343/241913 